\title{
Die jungen Wilden brauchen Führung: Treiber von Innovativität und Zufriedenheit im Arbeitsumfeld innovationsferner Branchen
}

\author{
Evelyn Kästner ${ }^{1}$ (D) Katja Rudolph ${ }^{2}$ \\ Angenommen: 3. Januar 2022 / Online publiziert: 24. Januar 2022 \\ (c) Der/die Autor(en) 2022
}

\section{Zusammenfassung}

Der vorliegende Beitrag in der Zeitschrift „Gruppe. Interaktion. Organisation.“ beantwortet die Frage, wie insbesondere Unternehmen in eher innovationsfernen Branchen wie der Versicherungs- und Energiebranche ihre Arbeitnehmer:innen über alle Altersstufen hinweg so unterstützen können, dass innovatives Verhalten am Arbeitsplatz optimal gefördert wird. Hintergrund ist, dass in den kommenden Jahren zwei Welten aufeinanderprallen: Unternehmen müssen innovativ sein, um im Wettbewerb zu bestehen. New Work als strategischer Ansatz ist dabei in aller Munde. Gleichzeitig steigt das Durchschnittsalter der Beschäftigten, weil qualifizierter Nachwuchs in vielen Bereichen fehlt.

In einer quantitativen Online-Befragung wurden auf Basis validierter Skalen Daten zur kreativitätsförderlichen Gestaltung des Arbeitsumfelds, zur Erwartungshaltung an New Work, zum innovativen Verhalten im Arbeitsumfeld und zur Zufriedenheit mit dem Ausschöpfungsgrad des eigenen Innovationspotenzials (Ausschöpfungszufriedenheit) erhoben. Die Antwortenden wurden mithilfe einer K-Means-Clusteranalyse nach Alter und innovativem Verhalten gruppiert, um im Folgenden die jüngeren Innovativen (,Junge Wilde“) mit den älteren Innovativen („Erfahrene Pioniere“) vergleichen zu können.

Ein durchaus überraschendes Ergebnis ist, dass „,die jungen Wilden“ mehr Führung und Struktur benötigen als „die erfahrenen Pioniere“. Da Innovativität signifikant mit der Ausschöpfungszufriedenheit korreliert, wurden ferner in linearen Regressionsanalysen die Treiber dieser Zufriedenheit im Arbeitsumfeld untersucht. Altersunabhängig führen regelmäßige Schulungen und Übungen zu mehr Zufriedenheit. Jedoch benötigen ,die jungen Wilden“ Führung und Unterstützung und kommen mit Projektgruppen weniger gut zurecht. „Die erfahrenen Pioniere“ hingegen benötigen in erster Linie den Willen und die Infrastruktur zum gegenseitigen Austausch. Die Ergebnisse legen daher eine altersabhängige Ausgestaltung des Arbeitsumfelds und des Führungsverhaltens nahe.

Schlüsselwörter New Work · Innovativität · Kreativität · Zufriedenheit · Demographischer Wandel · Employer Branding

\section{The young savages need leadership: drivers of innovativeness and satisfaction in the work environment of innovation-deficient industries}

\section{Abstract}

This article in the journal "Gruppe. Interaction. Organisation." answers the question of how companies in sectors that tend to be less innovative (e.g. insurance and energy sectors) can foster their employees' innovative behaviour across all age groups. This is of high importance since companies have to be innovative in order to survive in competition; New Work as a strategic approach is on everyone's lips. At the same time, the average age of employees is rising because qualified young people are lacking in many areas.

Prof. Dr. Evelyn Kästner e.kaestner@macromedia.de

Prof. Dr. Katja Rudolph katja.rudolph@hs-merseburg.de
Professur für Medienmanagement \& Marketing, Hochschule Macromedia, Leipzig (Sachsen), Deutschland

2 Professur für Allgemeine BWL, Marketing \& E-Business, Hochschule Merseburg, Merseburg (Sachsen-Anhalt), Deutschland 
A quantitative online survey based on validated scales was used to collect data on the design of a creativity-enhancing work environment, on expectations of New Work, on innovative behaviour in the working environment and on satisfaction with the degree of utilisation of one's own innovation potential (utilisation satisfaction). The respondents were grouped by age and innovative behaviour with the help of a K-Means cluster analysis in order to be able to compare the younger innovators ("young savages") with the older innovators ("experienced pioneers").

A surprising result is that "the young savages" need more guidance and structure than "the experienced pioneers". Since innovativeness correlates significantly with utilisation satisfaction, linear regression analyses were also used to examine the drivers of this satisfaction in the work environment. Regardless of age, regular training and exercises lead to more satisfaction. However, the "young savages" also need guidance and support and are less able to cope with project groups. The "experienced pioneers", on the other hand, primarily need the will and the infrastructure for mutual exchange. The results therefore suggest an age-dependent design of the working environment and leadership behaviour.

Keywords New Work $\cdot$ Innovativeness $\cdot$ Creativity $\cdot$ Satisfaction $\cdot$ Demographic change $\cdot$ Employer branding

\section{Einleitung}

Innovationen sind die treibende Kraft und die Zukunft des Wirtschaftsstandorts Deutschland. Auf Unternehmensebene helfen sie dabei, Unternehmensziele zu erreichen, Gewinn und Marktanteile zu steigern, Kund:innenbedürfnisse zu befriedigen und neue Kaufimpulse zu generieren (Segler 2000). Doch eine außergewöhnliche Innovation von heute verliert am nächsten Tag bereits an Wert, und Unternehmen müssen permanent innovativ sein, um im Wettbewerb langfristig zu bestehen. Organisationen sehen sich deshalb einem gewaltigen Innovationsdruck ausgesetzt. Aus diesem Grund ist Innovativität längst nicht mehr nur ein Nice-toHave, sondern wird branchenübergreifend immer mehr zum elementaren Bestandteil der Unternehmens-DNA (Rammer 2021, S. 6f.).

Da jede Innovativität im Unternehmen bei der Kreativität einzelner Menschen oder Teams beginnt (Amabile et al. 1996, S. 1155), rücken die Mitarbeiter:innen und ihre innovativen Verhaltensweisen immer stärker in den Blickpunkt des Interesses. Der Ansatz der sogenannten New Work soll dabei helfen, ein Arbeitsumfeld zu gestalten, in dem die Wünsche der Mitarbeiter:innen nach Autonomie bei der zeitlichen und räumlichen Arbeitsgestaltung, Agilität, Selbst- und Mitbestimmung durch flache Hierarchien, agile Teams oder Co-Working-Bereiche erfüllt werden (Grüling 2019), um das kreative Potenzial eines und einer Jeden optimal auszuschöpfen (Kästner 2009, S. 13). Die Etablierung solcher Strukturen und Mindsets funktioniert in einigen sehr innovationslastigen Branchen gut, stellt Branchen, bei denen Innovativität nur auf den zweiten Blick Relevanz hat, jedoch vor große Herausforderungen. Derartige Branchen sind laut ZEW Branchenreport Innovationen (2021) des Leibniz-Zentrums für Europäische Wirtschaftsforschung (ZEW) beispielsweise die Energie- wie auch die Versicherungsbranche, in denen die Innovationsleistung im Vergleich zur deutschen Gesamtwirtschaft bes- tenfalls durchschnittlich, wenn nicht gar unterdurchschnittlich ist.

Gleichzeitig besteht branchenübergreifend seit mittlerweile zwei Jahrzehnten ein verstärkter Kampf um qualifizierte Angestellte, weil die sog. Baby-Boomer - also Mitarbeitendenkohorten aus den geburtenstarken 60er und 70er-Jahren - vermehrt den Arbeitsmarkt verlassen (GeisThöne 2021, S. 3). Dabei kommt dem Aufbau einer Arbeitgebermarke (Employer Brand) eine große Bedeutung $\mathrm{zu}$ : Unternehmen versprechen potenziellen wie auch aktuellen Arbeitnehmer:innen kommunikativ Wertschätzung und Entwicklungsräume, finanzielle Benefits und sonstige Nutzenvorteile, um sich vom Wettbewerb, der ähnliche Arbeitsplätze anbietet, abzuheben (Melde und Benz 2014, S. 6).

Erschwerend kommt hinzu, dass das Durchschnittsalter des verbleibenden Mitarbeitendenstamms in vielen Branchen kontinuierlich ansteigt, weil wegen des demographischen Wandels qualifizierter Nachwuchs fehlt (BIB 2019; Volkholz 2005, S. 47ff.). Mit Blick auf Erkenntnisse der Alternsforschung, wonach die kognitive Leistungsfähigkeit ab einem Alter +50 kontinuierlich abnimmt (Reischies und Lindenberger 2010), stellt sich die Frage, wie Unternehmen eine auf mittelfristige Sicht schrumpfende und vor allem älter werdende Belegschaft dabei unterstützen können, das vorhandene Erfahrungswissen in einer förderlichen Arbeitsumgebung in innovative Verhaltensweisen zu überführen (Noefer et al. 2009).

Zielsetzung des Beitrages ist es deshalb, diejenigen Treiber von Innovativität im Arbeitsumfeld zu identifizieren, die die Belegschaft von Unternehmen in eher unternehmensfernen Branchen unabhängig vom Alter der Mitarbeitenden zu innovativen Verhaltensweisen führen. Dabei stehen folgende Forschungsfragen im Vordergrund:

- F1: Wie können Unternehmen in eher innovationsfernen Branchen ihre Mitarbeitenden über alle Altersstufen hin- 
weg so unterstützen, dass innovatives Verhalten am Arbeitsplatz optimal gefördert wird?

- F2: Wie kann es gelingen, die eigene Belegschaft über alle Altersstufen hinweg zufriedener zu machen, damit sie im Kampf um Talente im Unternehmen bleibt?

Zur Beantwortung dieser Fragen sollen zunächst die theoretischen Grundlagen für eine innovationsförderliche Arbeitsumgebung, der Zusammenhang zwischen Zufriedenheit und Innovativität und der daraus abgeleitete Bezugsrahmen des Beitrags dargestellt werden. Nachfolgend werden die Ergebnisse einer standardisierten Onlinebefragung vorgestellt, welche die Elemente der Unternehmenskultur (das Wollen von Innovation) und der Unternehmensstruktur (das Dürfen von Innovation) vor dem Hintergrund des Kreativitätspotenzials des Individuums (das Können von Innovation) untersucht haben.

\section{Theoretischer Hintergrund}

\subsection{Das Können von Innovation: Individuelle Kreativität als Ausgangsbasis von Innovativität}

„Die Kreativität ist der Ursprung, aus dem [...] neue Innovationen geboren werden" (Segler 2000, S. 77), ohne Kreativität gibt es keine Innovation (Brem 2016, S. 87). Mit Kreativität ist dabei eine geistige Ressource gemeint, die grundsätzlich bei jedem Individuum zu finden ist und einen schöpferischen Denkprozess umfasst ,[...], bei dem Erfahrungen der Vergangenheit kombiniert und wieder kombiniert werden, oftmals mit geistigen Verzerrungen, in einer solchen Art und Weise, dass man mit neuen Dingen, neuen Mustern, neuen Formen und Konfigurationen hervorkommt" (Winkelhofer 2006, S. 9). Dabei ist zwischen dem Kreativitätspotenzial, das in unterschiedlichem Maße in jedem Menschen gegeben ist (Gough 1979), und der tatsächlich sichtbaren Kreativität zu unterscheiden, die sich in Abhängigkeit von individuellen Gegebenheiten und Umfeld ganz unterschiedlich äußern kann.

Bei den individuellen Gegebenheiten spielen insbesondere die Intelligenz, das Alter, Emotionen und motivationale Komponenten eine Rolle. Unter Intelligenz soll hier ,das gesamte multifaktorielle Gebilde menschlicher kognitiver Fähigkeiten“ (Kästner 2009, S. 62) verstanden werden, zu dem auch das im Laufe des Lebens erworbene Wissen sowie die generelle Denkfähigkeit gehören. Auch wenn die Ergebnislage zum Zusammenhang zwischen Intelligenz und Kreativität sehr divers ist (vgl. dazu ausführlich Kästner 2009, S. 59ff.), lässt sich zusammenfassen, dass
- Wissen die Grundlage für Kreativität darstellt, zu viel Fachwissen den Assoziationshintergrund allerdings einengt,

- gemessen am Intelligenzquotienten (IQ) eine Mindestintelligenz notwendig ist, jenseits eines IQ von 120 jedoch keine Steigerung der kreativen Leistungen mehr erkennbar ist,

- divergente Denkprozesse, die darauf ausgerichtet sind, mehrere alternative Lösungen für Problemstellungen zu entwickeln, positiv mit Kreativität korrelieren.

Im Hinblick auf Wissen und Denkprozesse spielt das Alter eine Rolle bei der individuellen kreativen Leistungsfähigkeit. Gemäß dem Zwei-Komponenten-Modell lebenslanger intellektueller Entwicklung (Baltes et al. 1999, S. 486ff.) erreicht die fluide Intelligenz, die Grundlage des divergenten Denkens ist, im Alter von etwa 30 Jahren ihren Höhepunkt und nimmt danach erst langsam, dann deutlich schneller kontinuierlich ab. Im Gegensatz dazu bleibt die erfahrungsbasierte kristalline Intelligenz auch im Alter stabil oder steigt mit den Jahren an.

Zum Einfluss von Emotionen auf Kreativität gibt es keine einheitlichen empirischen Erkenntnisse (vgl. dazu ausführlich Kästner 2009, S. 79ff.). Emotionen sollen hier als eine kognitiv interpretierte physiologische Erregung verstanden werden, die eine emotional geprägte Verhaltensweise - einen Affekt - nach sich zieht (Kleinginna und Kleinginna 1981, S. 349). Der Einfluss des Affekts auf kreatives Handeln ist abhängig von Stärke und Qualität (positiv vs. negativ) der Emotion. Eine generell gültige, unidirektionale Kausalität zwischen Emotion und Kreativität ist also nicht belegt (Kästner 2009, S. 93f.). Dennoch ist die unternehmensseitige Steuerung des emotionalen Klimas am Arbeitsplatz von Relevanz für kreatives Verhalten. Denn die schwankenden emotionalen Stimmungen im Arbeitsumfeld - das von eher schwachen emotionalen Bindungen geprägt ist (Ruef 2002, S. 429ff.) - können das Handeln von Mitarbeitenden in die eine wie die andere Richtung beeinflussen (Barsade und Gibson 1998, S. 88ff.). Eng mit dem Affekt verbunden ist auch die Zufriedenheit von Mitarbeitenden, also die Erfüllung ihrer Erwartungshaltung. Zahlreiche Studien belegen einen unidirektionalen Zusammenhang: Zufriedenheit und kreatives Verhalten korrelieren positiv, wobei das Arbeitsumfeld wiederum die Zufriedenheit beeinflusst (Janssen und Van Yperen 2004, S. 376ff.; Judge und Ilies 2004, S. 665ff.). Im Umkehrschluss bedeutet dies, dass Unternehmen, die es nicht schaffen, das vorhandene Kreativitätspotenzial auszuschöpfen, mit einer geringeren Zufriedenheit wie auch Kreativität rechnen müssen (Shalley et al. 2000, S. 219ff.).

Hinsichtlich motivationaler Komponenten ist zwischen intrinsischer und extrinsischer Motivation zu unterscheiden. Bei intrinsisch motiviertem Handeln erfolgt das Ver- 
halten aus eigenem, innerem Antrieb (etwa zur Befriedigung des Selbstverwirklichungsbedürfnisses oder zum Erreichen eines Flow-Zustands) (Collins und Amabile 2006, S. 300f.), während extrinsisch motiviertes Handeln durch Anreize kontrolliert wird, die nicht Teil der eigenen Handlung sind (etwa Belohnungen, Restriktionen, Bewertungen) (Eisenberger und Cameron 1996, S. 1154). Die empirische Erkenntnislage zeigt wenig überraschend sehr deutlich einen positiven Zusammenhang zwischen intrinsischer Motivation und kreativem Verhalten (Csikszentmihalyi 1988, S. 167), weist für extrinsische Motivation aber verschiedene Ergebnisse auf (vgl. ausführlich Kästner 2009, S. 96ff.):

- Werden extrinsische Einflüsse als kontrollierend empfunden (bspw. personen- statt arbeitsbezogenes Feedback des bzw. der Vorgesetzten), haben sie einen negativen Einfluss auf die individuelle Kreativität, auch wenn die individuelle Produktivität dadurch steigen kann (Shalley 1995, S. 491ff.).

- Sind extrinsische Einflüsse dabei hilfreich, die eigene Kompetenz- und Selbstbestimmungswahrnehmung zu unterstützen, indem konstruktive Verbesserungsvorschläge oder Zeit- und Denkfreiräume geboten werden, hat dies einen positiven Effekt auf kreative Leistungen (Collins und Amabile 2006, S. 304f.).

Wie oben bereits beschrieben, ist Kreativität Voraussetzung für Innovation. Innovation wird hier verstanden als die erfolgreiche Umsetzung und Vermarktung von individuellen kreativen Ideen (Amabile 1988, S. 126; Fuglsang 2008, S. 6). Dabei kommt den Mitarbeitenden mit ihrer Kreativität in jeder einzelnen Phase des Innovationsprozesses eine wichtige Bedeutung zu (vgl. dazu überblickshaft Kästner 2009, S. 51ff.), weil für die Innovativität eines Unternehmens nicht nur Produktinnovationen, sondern ebenso Prozess-, Führungs- oder Marketinginnovation benötigt werden, die mithin in allen Innovationsphasen kreativer Verhaltensweisen bedürfen (Higgins und Wiese 1996, S. 12ff.). Diese Erkenntnis macht für die nachfolgende empirische Untersuchung eine produktunabhängige Betrachtung von Innovativität nötig, die sich auf innovative Verhaltensmuster im Arbeitsumfeld stützt.

\subsection{Das Wollen und Dürfen von Innovation: Innovationsfreundliche Unternehmenskultur, innovationsförderliche Strukturen \& New Work}

Individuelle Kreativität ist ein notwendiger, nicht jedoch hinreichender Faktor zur Ermöglichung von Innovativität (Carayannis und Gonzalez 2003, S. 159). Denn interessanterweise führt die angeborene Ressource von Kreativität, das Kreativitätspotenzial, nicht notwendigerweise auch zu innovativen Verhaltensweisen. Wie bereits bei der Zufriedenheit als individuelle Determinante von Kreativität kurz angerissen, braucht es ein Umfeld, das das vorhandene kreative Potenzial ausschöpft, damit daraus Innovationen werden (Oldham und Cummings 1996, S. 609ff.).

Innovativität muss im Unternehmen gewollt und fest in der Unternehmenskultur verankert sein: im Willen zur Förderung, Motivation und emotionalen Bindung jedes und jeder Mitarbeitenden. Dieses „Wollen“ von Innovativität ist Voraussetzung dafür, dass auch Strukturen geschaffen werden, in denen Mitarbeitende Innovativität leben ,dürfen“" (vgl. Disselkamp 2012, S. 73).

$\mathrm{Zu}$ einer in diesem Sinne innovationsfreundlichen Unternehmenskultur zählen ein unterstützender Führungsstil ebenso wie flache Hierarchien, ein bewusster Kommunikations- und Wissenstransfer, ein emotional positives Klima, ein motivierendes Anreizsystem und Prozesstrainings (vgl. dazu ausführlich Kästner 2009, S. 111ff.) sowie psychologische Sicherheit (vgl. Edmondson 2018, S. 16) - also jene Umfeldkomponenten, die die oben beschriebenen individuellen Determinanten von Kreativität unternehmensseitig unterstützen. Eine innovationsfreundliche Unternehmenskultur erhöht nach einer Untersuchung von Ernst (2003) signifikant den Innovationserfolg (S. 39). Dafür werden Freiräume zum Innovieren benötigt (ebd.), die ihrerseits von einem Betriebsklima abhängen, das etwa das Teilen von Wissen begünstigt und die Angst vor Fehlern reduziert (Disselkamp 2012, S. 75). Darüber hinaus sind offene, hierarchieübergreifende Kommunikationswege und klare Regelungen, die es Mitarbeitenden aller Hierarchiestufen ermöglichen, zu innovieren, relevante Faktoren einer innovationsfreundlichen Unternehmenskultur, die Innovationen „will“ und damit die Basis des „Dürfens“ liefert (ebd., S. 76f.). Weiterhin zählen Handlungsspielräume der Mitarbeitenden, Konfliktbewusstsein, die Bereitschaft, auch externe Kooperationen einzugehen sowie ein Personalmanagement, das kreative Leistungen fördert und anerkennt, zu den Innovationsfreiräumen (ebd., S. 77.).

Neben kulturellen sind v.a. auch strukturelle Aspekte in Unternehmen dafür verantwortlich, dass Mitarbeitende tatsächlich (nicht) innovieren „dürfen“. Innovationsförderliche Strukturen umfassen etwa Aspekte wie die Möglichkeit zum agilen Arbeiten, Inspiration durch ein kreatives Arbeitsumfeld oder Anreize z. B. durch Innovationswettbewerbe/Preise (BWG AG o.J.). Dazu zählt auch das Verständnis, Innovationen nicht auf einzelne Abteilungen zu beschränken oder sogar unternehmensübergreifend voranzutreiben und die entsprechenden Austauschformate dafür zur Verfügung zu stellen (Disselkamp 2012, S. 80).

Eine innovationsfreundliche Unternehmenskultur - das „Wollen“ von Innovativität - und eine innovationsfreundliche Unternehmensstruktur, die das „Dürfen“ von Innovation tatsächlich ermöglicht, gehen Hand in Hand und sind, wie die Ausführungen oben zeigen, nicht immer trennscharf. Das Konzept „New Work“ greift beide Voraussetzungen 
für Innovativität auf und verwebt sie zu einer Unternehmensphilosophie, in welcher sich der Mensch als Ursprung aller Innovationen in einer flexiblen Arbeitsumgebung bestmöglich entfalten kann (Grüling 2019, S. 9ff.). Der Begriff New Work wurde von dem Sozialphilosophen Frithjof Bergmann geprägt, der das Konstrukt aus einem gesellschaftspolitischen Kontext heraus betrachtet und einen alternativen Ansatz zur Lohnarbeit entwirft, welchen er als „Neue Arbeit" bezeichnet (Bergmann 2004). Bergmann hatte dabei eine konkrete Form der Arbeit im Sinne, von der die heutige Verwendung des Begriffs New Work abweicht. Eine einheitliche Definition des Begriffes gibt es nicht, die Forschung hierzu steht noch an den Anfängen (ebd, S. 1). Heute versteht man unter New Work die ,grundlegende und nachhaltige Veränderung der Arbeitswelt" (Hackl et al. 2017, S. 3), wozu entsprechende Initiativen und Maßnahmen wie z.B. moderne Raumkonzepte und flexible Arbeitszeiten gehören.

\section{Methodisches Vorgehen}

\subsection{Entwicklung eines Bezugsrahmens}

Innovativität kann im Unternehmen nur dort entstehen, wo kreatives Potenzial, innovationsfreundliche Unternehmenskultur und innovationsförderliche Strukturen aufeinandertreffen (Woodman et al. 1993, S. 294). Dabei ist jedem und jeder Mitarbeitenden ein individuelles Kreativitätspotenzial inhärent, ebenso wie eine solide erlernte Wissensbasis, die jedoch insbesondere erst durch einen bewussten Wissens- und Erfahrungsaustausch zu neuartigen Assoziationen und kreativen Ideen führt. All dies benötigt eine Unternehmenskultur, in der ein unterstützender Führungsstil und hierarchieübergreifende Kommunikationsstrukturen gelebt werden, die divergentes Denken fördert und fordert und auf diese Weise den Willen demonstriert, dass kreatives Verhalten wertgeschätzt wird. Ein derartige innovationsfreundliche Unternehmenskultur lässt sich strukturell bspw. mithilfe von Konzepten der New Work umsetzen, die individuelle Freiräume, Mitspracherecht und vor allem physische und geistige Austauschräume schafft. Auf diese Weise kann unter den Mitarbeitenden ein positives emotionales Klima entstehen, in dem sie mit der Ausschöpfung ihres individuellen Potenzials zufrieden sind, was ebenfalls zu einer Innovativitätssteigerung führen kann. Mit Blick auf die alternde Belegschaft ist darüber hinaus davon auszugehen, dass trotz sinkender fluider Intelligenz im Alter das Erfahrungswissen in Kombination mit einer innovationsförderlichen Arbeitsumgebung und Unternehmenskultur dennoch zu innovativen Verhaltensweisen führen kann (Noefer et al. 2009).

Die postulierten Zusammenhänge sind in Abb. 1 im Bezugsrahmen der nachfolgenden empirischen Untersuchung

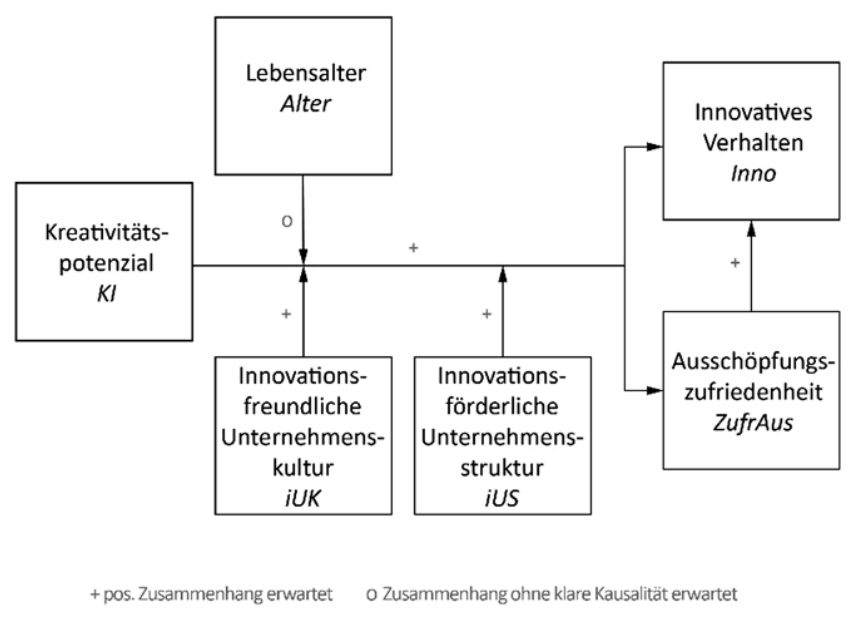

Abb. 1 Bezugsrahmen zur Darstellung der Einflussfaktoren auf innovative Verhaltensweisen im Arbeitsumfeld. (Quelle: Eigene Darstellung)

zusammengefasst. Aus ihm lassen sich - in Zusammenhang mit den in der Einleitung aufgestellten Forschungsfragen folgende zu prüfende Hypothesen ableiten:

- H-F1-a: Je höher das Kreativitätspotenzial von Mitarbeitenden ist, desto stärker zeigen sie innovative Verhaltensweisen.

- H-F1-b: Trifft ein hohes Kreativitätspotenzial auf eine innovationsfreundliche Unternehmenskultur, hat dies einen positiven Einfluss auf innovative Verhaltensweisen.

- H-F1-c: Trifft ein hohes Kreativitätspotenzial auf eine innovationsförderliche Unternehmensstruktur, hat dies einen positiven Einfluss auf innovative Verhaltensweisen.

- H-F1-d: Wie stark innovationsfreundliche Unternehmenskultur und innovationsförderliche Unternehmensstruktur zu einer Ausschöpfung des individuellen Kreativitätspotenzials führen, hängt vom Alter der Mitarbeitenden $a b$.

- H-F2-a: Je höher die Zufriedenheit mit der Ausschöpfung der eigenen Innovativität (Ausschöpfungszufriedenheit) eingeschätzt wird, desto stärker zeigen Mitarbeitende innovative Verhaltensweisen.

- H-F2-b: Je besser innovationsfreundliche Unternehmenskultur und innovationsförderliche Unternehmensstruktur das individuelle Kreativitätspotenzial ausschöpfen, desto höher ist auch die empfundene Ausschöpfungszufriedenheit.

- H-F2-c: Die Ausschöpfungszufriedenheit beeinflusst den Effekt, den innovationsfreundliche Unternehmenskultur und innovationsförderliche Unternehmensstruktur bei der Überführung bestehenden Kreativitätspotenzials in innovatives Verhalten haben, positiv. 


\subsection{Die Energie- und Versicherungsbranche als eher innovationsferne Grundgesamtheit}

Es gibt zahlreiche empirische Belege dafür, dass Innovativität in Unternehmen branchenübergreifend immer mehr zum elementaren Bestandteil der Unternehmens-DNA wird (Rammer 2021, S. 6f.). Das Bundesministerium für Bildung und Forschung (BMBF 2020) belegt diesen Trend mit stetig steigenden bundesweiten Ausgaben für Forschung und Entwicklung. Dennoch gibt es auch Branchen, bei denen die Innovationsleistung vergleichsweise geringer ist und entsprechend unterhalb des bundesdeutschen Durchschnitts liegt, wie dies in der Energie- sowie der Versicherungsbranche der Fall ist (ZEW 2021).

Für eine Untersuchung der Treiber von Innovativität und Zufriedenheit im Arbeitsumfeld sind solche als eher innovationsfern zu klassifizierenden Branchen besonders geeignet, weil hier unternehmensspezifische Unterschiede hinsichtlich der innovationsfreundlichen Kulturen und innovationsförderlichen Strukturen noch am ehesten beobachtbar sind. Zudem haben für diese Branchen mögliche Handlungsempfehlungen eine besondere Relevanz, wenn es darum geht, die eigene Innovationsfähigkeit zu steigern. Deshalb beschränkt die vorliegende Untersuchung sich hauptsächlich auf die Energie- und Versicherungsbranche, welche im Vergleich zu anderen Branchen geringe Innovationsausgaben sowie eine geringe Innovationsintensität aufweisen (ZEW 2021, S. 5).

Die Energiebranche wird in dieser Studie als Gesamtheit der Unternehmen in Deutschland in den Tätigkeitsgebieten Elektrizitäts-, Gas-, Wärme- und Kälte- sowie Wasserversorgung gemäß der Definition des statistischen Bundesamtes verstanden und bestand Ende 2020 aus ca. 2300 Unternehmen mit mehr als 20 Beschäftigten (Statistisches Bundesamt 2021). Zu diesem Zeitpunkt beschäftigte die Branche knapp 260.000 Personen, wovon ca. $78 \%$ in der Elektrizitätsversorgung tätig waren (ebd.). Die Branche erzielte dabei Ende 2020 einen Jahresumsatz von knapp 590 Mrd. $€$ (Statistisches Bundesamt 2021a). Wie viele andere Branchen bekommt auch die Energiebranche den Fachkräftemangel zunehmend zu spüren (Preuß 2021).

Die Versicherungsbranche wird für diese Studie verstanden als Unternehmen der Bereiche Lebens-, Kranken-, Schaden- und Unfallversicherung sowie Rückversicherer, Pensionskassen und Sterbekassen unter deutscher Bundesaufsicht (GDV 2020, S. 15). Ende 2019 zählte die Branche in Deutschland 530 Unternehmen, wovon ca. 200 Schadens- oder Unfallversicherer und ca. 80 Lebensversicherer waren (ebd.). 2018 erzielte die Branche 293 Mrd. $€$ Umsatz (ebd., S. 39) und beschäftigte Ende 2019 ca. 291.000 Arbeitnehmer:innen sowie zusätzlich knapp 200.000 selbstständige Versicherungsvermittler/ -berater:innen (ebd., S. 15). Wie die Energiebranche hat auch die Versicherungsbranche mit Fachkräftemangel zu kämpfen (AGV 2020, S. 29). Gleichzeitig steigt der Altersdurchschnitt der Beschäftigten, sodass die am häufigsten vertretene Altersgruppe mittlerweile die der über MittFünziger ist (AGV o.J.).

\subsection{Erhebungsinstrument und Stichprobe}

Als Erhebungsinstrument wurde eine standardisierte Befragung mit größtenteils geschlossenen und wenigen offenen Fragen entwickelt, die sich - wo immer es möglich war auf vorhandene Skalen stützte. Neben üblichen soziodemographischen Angaben zu Branchenzugehörigkeit, Unternehmensgröße und Position im Unternehmen wurde das Alter offen abgefragt, um mit einem metrischen Skalenniveau rechnen zu können. Das Kreativitätspotenzial wurde über die Creative Personality Scale von Gough (1979) abgefragt (in einer deutschen Übersetzung von Kästner 2009, S. 166ff.), nach der sich das individuelle Kreativitätspotenzial aus zeitlich verhältnismäßig stabilen kreativitäts(ir-)relevanten Persönlichkeitseigenschaften ableiten lässt (die Skalenreliabilität ist mit Cronbach's Alpha $=0,629$ für kreativitätsrelevante Eigenschaften, Cronbach's Alpha =0,510 für kreativitätshemmende Eigenschaften und Cronbach's Alpha $=0,523$ für die Gesamtskala gerade noch akzeptabel). Die Komponenten einer innovationsfreundlichen Unternehmenskultur sind einer repräsentativen Erhebung von Kästner (2009, S. 168ff.; Cronbach's Alpha 0,908) entnommen, die Skala zur Erfassung der strukturellen Gegebenheiten eines innovationsförderlichen Arbeitsumfelds basiert auf Hays et al. (2016, S. 10, Cronbach's Alpha=0,831). Einzig zur Erfassung der Komponenten von New Work wurde in Zusammenarbeit mit New Work-Experten eine eigene Skala entwickelt, die Items zur Arbeitsplatzgestaltung, zu Austauschmöglichkeiten, zur Organisationsstruktur sowie zu individuellen Förderangeboten für Mitarbeitende abfragte und in Folgestudien zu validieren ist, mit einem Cronbach's Alpha $=0,832$ aber bereits eine sehr gute interne Skalenkonsistenz aufweist. Für die Erhebung innovativer Verhaltensweisen im Arbeitsumfeld wurde auf eine Skala von Sarges (2013, S. 465, Cronbach's Alpha=0,842) zurückgegriffen. Die Zufriedenheit wurde als doppeltes Globalkonstrukt mit vierstufiger Ratingskala abgefragt: einmal als generelle Zufriedenheit im Arbeitsumfeld sowie als tatsächlich kreativitätsrelevante Zufriedenheit mit der selbstempfundenen Ausschöpfung des eigenen kreativen Potenzials (Ausschöpfungszufriedenheit). Ersteres wurde nur abgefragt, um für die Ausschöpfungszufriedenheit Effekte der allgemeinen Zufriedenheitseinschätzung zu minimieren.

Der Online-Fragebogen wurde im Zeitraum vom 22. September 2020 bis 30. November 2020 über Verteiler der Versicherungsforen Leipzig (ca. 1400 potenzielle Befragungsteilnehmer:innen) und der Energieforen Leipzig 
Tab. 1 Demographische Charakteristika des Datensatzes. (Quelle: Eigene Darstellung)

\begin{tabular}{lllr}
\hline Geschlechtszugehörigkeit & & Branchenzugehörigkeit & \\
Männlich & $45,1 \%$ & Energiewirtschaft & $43,7 \%$ \\
Weiblich & $53,5 \%$ & Assekuranz (Versicherungen) & $23,9 \%$ \\
Divers & $1,4 \%$ & Bildung \& Forschung & $9,9 \%$ \\
Alter & & Marketing \& Werbung & $8,5 \%$ \\
$\varnothing$ & 39,7 Jahre & Sonstige Dienstleistungen & $5,6 \%$ \\
Unternehmensgröße & & Beratung & $4,2 \%$ \\
KMU & $31,4 \%$ & Wirtschaft und Finanzen & $4,2 \%$ \\
Große Unternehmen & $27,1 \%$ & Kreativitätspotenzial & \\
Sehr große Unternehmen \& Konzerne & $41,5 \%$ & $\varnothing$ & 3,72 \\
& & Innovatives Verhalten im Arbeitsumfeld \\
& & $\varnothing$ & 4,00 \\
\hline
\end{tabular}

(1388 potenzielle Befragungsteilnehmer:innen) sowie über private Verteiler der Autorinnen und Praxispartner bei den Versicherungsforen Leipzig sowie Energieforen Leipzig (ca. 200 potenzielle Befragungsteilnehmer:innen) ins Feld gegeben. Die Rücklaufquote lag bei $2,4 \%$ und brachte 71 verwertbare Datensätze. Die Befragung ist mithin nicht repräsentativ, zeigt jedoch signifikante Ergebnisse, so dass dennoch von einer grundsätzlichen Gültigkeit der Aussagen ausgegangen werden kann (Cook et al. 2000, S. 821f.). Die geringe Rücklaufquote wird auf das spezifische Thema zurückgeführt, das nur für einen Teil der angeschriebenen Personen relevant war. Die Datenanalyse erfolgte mit SPSS 25 mit einer Erweiterung für Moderations- und Mediationsanalysen durch PROCESS 4.0 (Hayes 2018).

Hinsichtlich der demographischen Charakteristika des Datensatzes (vgl. Tab. 1) ist festzustellen, dass eine leichte Mehrheit der Befragten angab, weiblichen Geschlechts zu sein. Im Durchschnitt waren die Befragten knapp 40 Jahre alt. Die Branchenzugehörigkeit entspricht den verwendeten Verteilern: ein knappes Viertel der Befragten arbeitet in der Versicherungsbranche, knapp $44 \%$ in der Energiebranche, so dass der Datensatz wie angestrebt einen Großteil an Befragten aus eher innovationsfernen Branchen beinhaltet. Entsprechend der EU-Klassifikation (Europäische Kommission 2015, S. 10) konnten $31 \%$ der Antworten kleinen und mittelständischen Unternehmen bis 249 Mitarbeitenden zugeordnet werden, die restlichen verteilen sich auf $27 \%$ groBe Unternehmen mit bis zu 999 Mitarbeitenden und $41 \%$ sehr große Unternehmen oder Konzerne mit 1000 und mehr Mitarbeitenden. Das durchschnittliche Kreativitätspotenzial der Mitarbeitenden ist, klassifiziert in 5 Klassen, mit einem Wert von 3,71 auf einer Skala von 1 (sehr geringes Kreativitätspotenzial) bis 5 (sehr hohes Kreativitätspotenzial) im Vergleich zu einer für die Gesamtbevölkerung repräsentativen Untersuchung (Kästner 2009; Wert 3,27) leicht überdurchschnittlich, der durchschnittliche Wert für das innovative Verhalten im Arbeitsumfeld, ebenfalls klassifiziert in fünf Klassen, liegt bei 4,00 (Skala 1 ,gar nicht innovativ“ bis 5 ,sehr innovativ“) und damit in einem hohen Bereich.

\section{Empirischer Befund}

\subsection{Varianz innerhalb der Hauptvariablen}

Bevor die multivariate Hypothesenprüfung erfolgen kann, sollen die Hauptvariablen Kreativitätspotenzial KI, innovatives Verhalten Inno, innovationsfreundliche Unternehmenskultur $i U K$ und innovationsförderliche Unternehmensstruktur $i U S$ kurz deskriptiv beschrieben werden. Wie in Tab. 2 ersichtlich wird, ist die Varianz innerhalb der Variablen, gemessen an der Skalenspannweite, eher gering, was als Bestätigung für die Homogenität innerhalb der Stichprobe interpretiert werden kann und bei den folgenden Berechnungen $\mathrm{zu}$ berücksichtigen ist.

\subsection{Kreativitätspotenzial, Alter, innovations- freundliche Unternehmenskultur und innovationsförderliche Unternehmensstruktur als Treiber von innovativem Verhalten}

Eine der Grundvoraussetzungen für innovatives Verhalten im Arbeitsumfeld ist das Kreativitätspotenzial der Mitarbeitenden. Eine lineare Regressionsanalyse des innovativen Verhaltens Inno in Abhängigkeit vom Kreativitätspotenzial $K I$ zeigt ein hoch signifikantes Gesamtmodell, $\mathrm{F}(1,69)=16,686, p<0,001$, mit einer mittleren bis hohen Varianzaufklärung von 19,5\%. Das Vorhandensein des Kreativitätspotenzials ist mithin ein wichtiger Treiber innovativen Verhaltens, Hypothese H-F1-a kann damit bestätigt werden.

Tab. 2 Varianz innerhalb der Variablen. (Quelle: Eigene Darstellung)

\begin{tabular}{lllllll}
\hline Variable & $\begin{array}{l}\text { Skalen- } \\
\text { spannweite }\end{array}$ & $N$ & Min & Max & $\begin{array}{l}\text { Mittel- } \\
\text { wert }\end{array}$ & $\begin{array}{l}\text { Standard- } \\
\text { abweichung }\end{array}$ \\
\hline KI & -12 bis $+18[30]$ & 71 & -2 & 13 & 8,20 & 2,755 \\
Inno & 16 bis 64 [49] & 71 & 40 & 64 & 50,45 & 5,684 \\
$i U K$ & 15 bis 60 [46] & 71 & 22 & 60 & 41,25 & 8,220 \\
$i U S$ & 0 bis 120 [121] & 71 & 12 & 78 & 40,80 & 15,504 \\
\hline
\end{tabular}


Wie jedoch in den theoretischen Grundlagen dargelegt, kommt der innovationsfreundlichen Unternehmenskultur $i U K$ eine wichtige Rolle bei der Ausschöpfung des Kreativitätspotenzials zu (Woodman et al. 1993, S. 294). Es wurde daher eine Moderationsanalyse durchgeführt, um zu bestimmen, ob die Interaktion zwischen Kreativitätspotenzial $K I$ und innovationsfreundlicher Unternehmenskultur $i U K$ innovatives Verhalten im Arbeitsumfeld Inno signifikant vorhersagt. Das Gesamtmodell war hoch signifikant, $\mathrm{F}(3,67)=11,8368, p<0,001$, mit einer recht hohen Varianzaufklärung von $24,07 \%$. Jedoch konnten die Ergebnisse keinen Moderationseffekt der innovationsfreundlichen Unternehmenskultur $i U K$ auf die Beziehung zwischen Kreativitätspotenzial $K I$ auf innovatives Verhalten Inno finden, $\Delta \mathrm{R}^{2}=0,04 \%, \mathrm{~F}(1,67)=0,0596, p=0,808$ bei $95 \%$ Konfidenzintervall $[-0,0653,0,0437]$. In eher innovationsfernen Branchen hat eine innovationsfreundliche Unternehmenskultur also keinen Einfluss darauf, ob aus einem kreativen Potenzial innovatives Verhalten wird. Hypothese H-F1-b wurde mithin widerlegt.

Nach den Ausführungen zum Können (Kreativitätspotenzial) und Wollen (Unternehmenskultur) von Innovativität sollen nun die Ergebnisse zum Dürfen von Innovativität vorgestellt werden: der innovationsfreundlichen Unternehmensstruktur. Dafür wurden die 20 Items der Eigenskala als Summenwert iUS zusammengefasst. Eine lineare Regressionsanalyse des innovativen Verhaltens Inno in Abhängigkeit von der innovationsfreundlichen Unternehmensstruktur $i U S$ zeigt ein signifikantes Gesamtmodell, $\mathrm{F}(1,69)=6,424$, $p=0,014$, mit einer geringen Varianzaufklärung von $8,5 \%$. Es gibt also einen kleinen direkten Effekt von innovationsförderlichen Angeboten auf das innovative Verhalten. Auch hier ist gemäß den theoretischen Ausführungen ein Moderationseffekt zu erwarten, bei dem das Vorhandensein innovationsförderlicher Strukturen die Ausschöpfung des Kreativitätspotenzials als innovatives Verhalten beeinflusst. Die durchgeführte Moderationsanalyse zeigt in einem hoch signifikanten Gesamtmodell mit $\mathrm{F}(3,67)=7,465$, $p<0,001$ und einer hohen Varianzaufklärung von 28,3\% einen schwach signifikanten Moderationseffekt, nach dem die innovationsfreundliche Unternehmensstruktur $i U S$ den direkten Effekt zwischen Kreativitätspotenzial KI und innovativem Verhalten Inno schwach signifikant moderiert, $\Delta \mathrm{R}^{2}=4,2 \%, \mathrm{~F}(1,67)=3,902, p=0,052$ bei einem Konfidenzintervall von $95 \%[-0,060,-0,008]$. Das Angebot von Strukturen, in denen Innovativität gelebt werden kann, hat also einen zusätzlichen, wenn auch kleinen Effekt auf die Ausschöpfung des Kreativitätspotenzials im Arbeitsumfeld. Hypothese H-F1-c kann bestätigt werden, wenngleich der ermittelte Effekt kleiner ist als erwartet.

Ein weiterer möglicher Einflussfaktor auf innovatives Verhalten ist das Alter, dessen Einflussqualität in der Literatur jedoch ambivalent gesehen wird. Viele Autoren be- legen, dass mit Rückgang der fluiden Intelligenz die Innovativität mit steigendem Alter zurückgeht. Gleichzeitig bleibt die erfahrungsbasierte kristalline Intelligenz auch im Alter stabil oder steigt mit den Jahren an. Mithilfe einer weiteren Moderationsanalyse sollte bestimmt werden, ob die Interaktion zwischen Kreativitätspotenzial $\mathrm{KI}$ und $\mathrm{Al}$ ter innovatives Verhalten im Arbeitsumfeld Inno signifikant vorhersagt. Das Gesamtmodell war erneut signifikant, $\mathrm{F}(3,66)=7,8159, p<0,001$, mit einer mittleren bis hohen Varianzaufklärung von $20,06 \%$. Jedoch besteht kein Moderationseffekt der Variablen Alter auf die Beziehung zwischen Kreativitätspotenzial $K I$ und innovativem Verhalten Inno: $\Delta \mathrm{R}^{2}=0,05 \%, \mathrm{~F}(1,66)=0,3795, p=0,540$ bei einem $95 \%$ Konfidenzintervall $[-0,0702,0,0191]$. Die Ausschöpfung des Kreativitätspotenzials im Arbeitsumfeld ist also nicht altersabhängig, Hypothese H-F1-d muss daher widerlegt werden.

Als erstes Zwischenergebnis lässt sich festhalten, dass nur das individuelle Kreativitätspotenzial einen hohen direkten Einfluss auf innovatives Verhalten im Arbeitsumfeld von eher innovationsfernen Branchen hat. Eine innovationsfreundliche Unternehmenskultur hat kaum einen Effekt, das Angebot eines innovationsförderlichen Umfeldes (Struktur, Angebote) hat zumindest einen kleinen direkten sowie einen moderierenden Effekt auf die Überführung des vorhandenen Potenzials in innovatives Verhalten. Das Alter beeinflusst innovatives Verhalten hingegen nicht.

\subsection{Ausschöpfungszufriedenheit als direkter und indirekter Treiber von innovativem Verhalten}

Ein weiterer Treiber von Innovativität ist die sogenannte Ausschöpfungszufriedenheit. Ihr zufolge steigt die Innovativität im Arbeitsumfeld, je zufriedener Arbeitnehmer und Arbeitnehmerinnen mit der Ausschöpfung ihres eigenen kreativen Potenzials im Arbeitsumfeld sind (Shalley et al. 2000, S. 215ff.). Eine einfache lineare Regression zeigt in einem schwach signifikanten Gesamtmodell, $F(1,69)=3,781, p=0,056$, mit einer geringen Varianzaufklärung von 5,2\% einen kleinen direkten Effekt der Ausschöpfungszufriedenheit ZufrAus auf innovatives Verhalten Inno. Nimmt man das Kreativitätspotenzial $K I$ in die Regressionsgleichung auf, erreichen in einem hoch signifikanten Gesamtmodell, F $(2,68)=10,982, p<0,001$, die beiden Variablen eine Varianzaufklärung des innovativen Verhaltens von hohen $24,4 \%$. Um zu prüfen, ob es einen zusätzlichen moderierenden Effekt der Ausschöpfungszufriedenheit ZufrAus auf den direkten Zusammenhang zwischen Kreativitätpotenzial KI und innovativem Verhalten Inno gibt - ob also die Verwandlung kreativen Potenzials in innovative Verhaltensweisen von der Ausschöpfungszufriedenheit beeinflusst wird - wurde auch hier eine Moderationsanalyse durchgeführt. Trotz signifikanten Gesamtmodells, 
$\mathrm{F}(3,67)=10,4685, p<0,001$, mit einer hohen Varianzerklärung von $25,82 \%$ liegt kein zusätzlicher Moderationseffekt vor $\left(\Delta \mathrm{R}^{2}=1,41 \%, \mathrm{~F}(1,67)=1,6615, p=0,202\right.$ bei einem $95 \%$ Konfidenzintervall $[-0,9609,0,1120])$. H-F2-a kann dennoch für einen kleinen direkten Effekt bestätigt werden.

Um zu überprüfen, ob die Ausschöpfungszufriedenheit ZufrAus den Effekt der innovationsfreundlichen Unternehmenskultur $i U K$ bzw. der innovationsförderlichen Unternehmensstruktur $i U S$ auf innovatives Verhalten Inno ganz oder partiell mediiert, wurden zwei Mediationsanalysen berechnet. Entsprechend den Vorgaben nach Baron und Kenny (1986) liegen bei beiden Modellen keine Mediationseffekte vor, weil entweder der direkte Effekt nicht signifikant war $(i U K)$ und/oder einer der beiden indirekten Pfade nicht signifikant war. Hypothese H-F2-c ist daher zu widerlegen. Auffällig im Mediationsmodell der innovationsförderlichen Unternehmensstruktur $i U S$ war jedoch der hoch signifikant $(p<0,001)$ positive indirekte Effekt zwischen $i U S$ und Ausschöpfungszufriedenheit, der sich auch in einer linearen Regression mit der Ausschöpfungszufriedenheit ZufrAus als abhängige und der innovationsförderlichen Unternehmensstruktur $i U S$ als unabhängige Variable bestätigt. Das hoch signifikante Gesamtmodell mit F $(1,69)=19,492, p<0,001$, kann $22 \%$ der Varianz der Ausschöpfungszufriedenheit erklären. Damit kann Hypothese H-F2-b teilweise bestätigt werden.

Zusammenfassend lässt sich aus den bisherigen Ergebnissen ableiten, dass im vorliegenden Gesamtdatensatz von Befragten aus vornehmlich eher innovationsfernen Branchen das Kreativitätspotenzial sowie konkrete Angebote zur Innovationsförderung, nicht jedoch die innovationsfreundliche Unternehmenskultur zu innovativem Verhalten und Ausschöpfungszufriedenheit führen. Zudem zeigen sich signifikante Zusammenhänge zwischen Ausschöpfungszufriedenheit und innovativem Verhalten.

Im Folgenden soll daher ganz konkret analysiert werden, woraus sich diese Ausschöpfungszufriedenheit als eine der Grundlagen für innovatives Verhalten speist. Bislang wurden die Zusammenhänge auf Basis von Summenwerten analysiert, um möglichst viel Informationsvarianz zu verwerten. Für die konkrete Ausgestaltung des Arbeitsumfeldes zur Förderung von Innovativität und Zufriedenheit braucht es jedoch einen detaillierten Blick auf einzelne gestaltbare Parameter. Dabei soll es darum gehen, für diejenigen Befragten, die tatsächlich innovatives Verhalten zeigen, die Arbeitsumgebung sowie die Treiber ihrer Zufriedenheit zu untersuchen. Mit Blick auf die Lebensrealität in Unternehmen - alternde Belegschaften und Kampf um hochqualifizierten Nachwuchs - wird dabei im Folgenden ein Split nach Alter vorgenommen.
Tab. 3 Beschreibung von drei Mitarbeitendenclustern anhand der Variablen „Alter“ und „Innovativität“. (Quelle: Eigene Darstellung)

\begin{tabular}{llll}
\hline & $\begin{array}{l}\text { „Die jungen } \\
\text { Wilden“ }\end{array}$ & $\begin{array}{l}\text { „Das Mittel- } \\
\text { maß“ }\end{array}$ & $\begin{array}{l}\text { „Die erfahrenen } \\
\text { Pioniere“ }\end{array}$ \\
\hline$n$ & 33 & 22 & 12 \\
Alters- $\varnothing$ & 32,97 & 41,46 & 50,74 \\
$\begin{array}{l}\text { Innovativität } \\
(16-64)\end{array}$ & 50,62 & 42,42 & 54,30 \\
$\begin{array}{l}\text { Geschlecht } \\
(\mathrm{m}|\mathrm{w}| \mathrm{d})\end{array}$ & $50 \% \mid 50 \%$ & $38,5 \% \mid$ & $39,1 \% \mid 60,9 \%$ \\
\hline
\end{tabular}

\subsection{Clusteranalyse nach Alter und Innovativität}

Um Treiber von innovativem Verhalten im Arbeitsumfeld in Abhängigkeit vom Alter zu identifizieren, wurden die Befragten mittels einer K-Means-Clusteranalyse (vgl. zum analytischen Vorgehen Bacher et al. 2010, S. 15) zunächst anhand der Variablen Alter (metrisch) und innovatives Verhalten Inno (metrischer Summenwert aus der formativen Summation aller Item-Bewertungen) in drei Gruppen klassifiziert. Sie lassen sich wie folgt beschreiben (vgl. Tab. 3):

- Gruppe 1 ist 32,97 Jahre alt und erreicht als Summenwert der Innovativität (Skala 16-64) einen Wert von 50,62 Punkten. Wir nennen diese Gruppe „Die jungen Wilden“.

- Gruppe 2 ist 41,46 Jahre alt und ist mit einem Summenwert der Innovativität von 42,42 nur mittelmäßig innovativ. Diese Gruppe nennen wir „Das Mittelmaß“.

- Gruppe 3 ist 50,74 Jahre alt und erreicht mit einer Innovativitätssumme von 54,30 einen sehr hohen Wert. Wir nennen diese Gruppe „Die erfahrenen Pioniere“.

Für die weiteren Analysen ist insbesondere der Vergleich zwischen den ,jungen Wilden“ und den „erfahrenen Pionieren" von Interesse: Maßnahmen, die über beide Altersgruppen zu Innovativität und Zufriedenheit führen, sind nicht altersspezifisch. Treten Unterschiede in den Ergebnissen auf, kann der Rückschluss gezogen werden, dass ältere Innovative in diesen Punkten ein anderes Arbeitsumfeld benötigen, um innovativ und zufrieden zu sein, als jüngere Innovative.

\subsection{Einflussfaktoren auf die Ausschöpfungszufriedenheit bei jüngeren und älteren Innovativen}

Zur Untersuchung der konkreten Einflussfaktoren der Ausschöpfungszufriedenheit werden lineare Regressionsanalysen zum Einfluss der innovationsfreundlichen Unternehmenskultur $i U K$ und der innovationsförderlichen Unternehmensstruktur $i U S$ auf die Ausschöpfungszufriedenheit $Z u$ frAus im Vergleich der Cluster ,die jungen Wilden“ und „die erfahrenen Pioniere“ vorgestellt. Damit soll die Frage beantwortet werden, wie eine Arbeitsumgebung gestaltet 
Tab. 4 Güte der Gesamtmodelle für die Regressionen iUK $\rightarrow$ ZufrAus. (Quelle: Eigene Darstellung)

\begin{tabular}{llll}
\hline Items $\mathrm{iuK}_{\mathrm{iUK}} \rightarrow$ ZufrAus & $\mathrm{R}^{2}$ & $\mathrm{~F}(\mathrm{df})$ & Sign \\
\hline "Die jungen Wilden“ & 0,681 & $2,561(15,18)$ & 0,030 \\
"Die erfahrenen Pioniere“ & 0,960 & $11,151(15,7)$ & 0,002 \\
\hline
\end{tabular}

sein muss, damit sie die Erwartungen der Mitarbeitenden erfüllt und diese mit der Ausschöpfung ihres kreativen Potenzials zufrieden sind. Als minimal erforderliches Signifikanzniveau wird wegen des erwartbar geringen Einzeleinflusses der Items im Folgenden mit einer Irrtumswahrscheinlichkeit von $<10 \%$ gerechnet $(p<0,100)$ (Pritschet et al. 2016).

Die Untersuchung des Einflusses einzelner Items der innovationsförderlichen Unternehmenskultur ergab zunächst für die beiden Cluster zwei signifikante Gesamtmodelle mit hoher erklärter Gesamtvarianz (vgl. Tab. 4).

Die Kollinearitätsdiagnostik war für ,die jungen Wilden“ unauffällig (alle Toleranzwerte $>0,1$ ). Bei den ,erfahrenen Pionieren" hingegen zeigten sich bei den Variablen „Die Vorgesetzten geben auf schnellstem Wege Feedback“, „Für die Lösung von Problemen arbeiten oft Kollegen unterschiedlicher Abteilungen zusammen“ und „Der Austausch über alle Führungsebenen hinweg ist unkompliziert" Toleranzwerte $<0,1$ - Hinweise auf eine starke Multikollinearität der Variablen, was auf die sehr hohe Skalenreliabilität (Cronbach's Alpha 0,908) zurückzuführen ist. Insbesondere „Die Vorgesetzten geben auf schnellstem Wege Feedback“ und „Der Austausch über alle Führungsebenen hinweg ist unkompliziert" korrelieren extrem hoch (zweiseitige Korrelation nach Pearson 0,941, $p<0,001)$. Die drei Variablen wurden daher aus der Regression für dieses Cluster ausgeschlossen. Wie in Tab. 5 dargestellt, zeigte sich im Ergebnis kaum eine Änderung der erklärten Varianz im zweiten Regressionsmodell, die Kollinearitätsdiagnostik war nun unauffällig.

Wie Tab. 6 zeigt, speist sich die Ausschöpfungszufriedenheit der jüngeren im Vergleich zu den älteren Innovativen aus wenigen konkreten Komponenten einer innovationsfreundlichen Unternehmenskultur. „Die jungen Wilden“ empfinden ihr Potenzial dann als ausgeschöpft, wenn sie Anleitung erhalten - durch Schulungen oder ihre Vorgesetzten. Die Zusammenstellung effektiver Projektgruppen durch die Vorgesetzen hat allerdings einen negativen Einfluss auf die Ausschöpfungszufriedenheit, mutmaßlich, weil

Tab. 5 Güte der Gesamtmodelle für die Regressionen iUK $\rightarrow$ ZufrAus mit angepasstem Modell für ,die erfahrenen Pioniere“ nach Kollinearitätsdiagnostik. (Quelle: Eigene Darstellung)

\begin{tabular}{llll}
\hline Items $\mathrm{iuK}_{\mathrm{i}} \rightarrow$ ZufrAus & $\mathrm{R}^{2}$ & $\mathrm{~F}(\mathrm{df})$ & Sign \\
\hline "Die jungen Wilden“ & 0,681 & $2,561(15,18)$ & 0,030 \\
„Die erfahrenen Pioniere“ & 0,830 & $4,060(12,10)$ & 0,017 \\
\hline
\end{tabular}

sich „die jungen Wilden“ dort nicht so entfalten können im Vergleich zu einer Konstellation, in der sie Teams selbst zusammenstellen oder alleine arbeiten können. „Die erfahrenen Pioniere" hingegen benötigen den Austausch mit anderen Kolleg:innen. Interessant ist, dass unabhängig vom Alter Innovative regelmäßige Schulungen und Übungen erwarten. Insgesamt ist der Einfluss der innovationsfreundlichen Unternehmenskultur auf die Ausschöpfungszufriedenheit auf Ebene der Einzelitems jedoch ebenso überschaubar wie der oben besprochene Einfluss auf innovative Verhaltensweisen.

Bei der innovationsförderlichen Unternehmensstruktur ermöglichte der Fragebogen die Angabe, dass strukturelle Items im eigenen Unternehmen nicht umgesetzt werden oder nicht bekannt sind. Durch listen- und paarweise Fallausschlüsse ist eine Regressionsanalyse mangels gültiger Fälle hier nicht möglich. Stattdessen wurden zweiseitige Korrelationen (nach Pearson) der Einzelitems mit der Ausschöpfungszufriedenheit gerechnet (vgl. Tab. 7).

In Bestätigung der Analyse der Treiber von Ausschöpfungszufriedenheit im Hinblick auf die Unternehmenskultur benötigen „die erfahrenen Pioniere“ bei den strukturellen Gegebenheiten vor allem Austauschformate und -flächen. „Die jungen Wilden“ hingegen sind dann zufrieden mit der Ausschöpfung ihres kreativen Potenzials, wenn sie fast das gesamte Spektrum des New-Work-Konzeptes nutzen können; angefangen von flexibler Arbeitsorganisation über Methoden und Strukturen des Ideenmanagements bis hin zu Austauschformaten.

Die Ergebnisse zum Einfluss einzelner Komponenten einer innovationsfreundlichen Unternehmenskultur und innovationsförderlichen Unternehmensstruktur sind ein weiterer deutlicher Beleg für Hypothese H-F2-b. Es lassen sich bei innovativen Mitarbeitenden klare Hinweise dafür finden, dass Komponenten innovationsfreundlicher Unternehmenskultur und einer innovationsförderlichen Unternehmensstruktur zu einer höheren Ausschöpfungszufriedenheit führen, und zwar abhängig vom Alter.

\subsection{Zusammenfassung des empirischen Befundes}

Mittels linearer Regressionsanalysen, Moderations- und Mediationsanalysen, Cluster- und Korrelationsanalysen wurden in diesem Beitrag die Treiber innovativen Verhaltens in eher innovationsfernen Branchen untersucht. Dabei wurde deutlich, dass das individuelle Kreativitätspotenzial, das einem Menschen individuell inhärent ist, auch im Arbeitsumfeld einen wichtigen Treiber innovativen Verhaltens darstellt. Entgegen sonstigen empirischen Untersuchungen zeigt sich jedoch, dass es keinen direkten Zusammenhang zwischen einer innovationsfreundlichen Unternehmenskultur und innovativem Verhalten gibt möglicherweise verhalten sich Mitarbeitende in innovati- 
Tab. 6 Clusterspezifische Einflüsse der Einzelitems innovationsfreundlicher Unternehmenskultur auf die Ausschöpfungszufriedenheit. (Quelle: Eigene Darstellung)

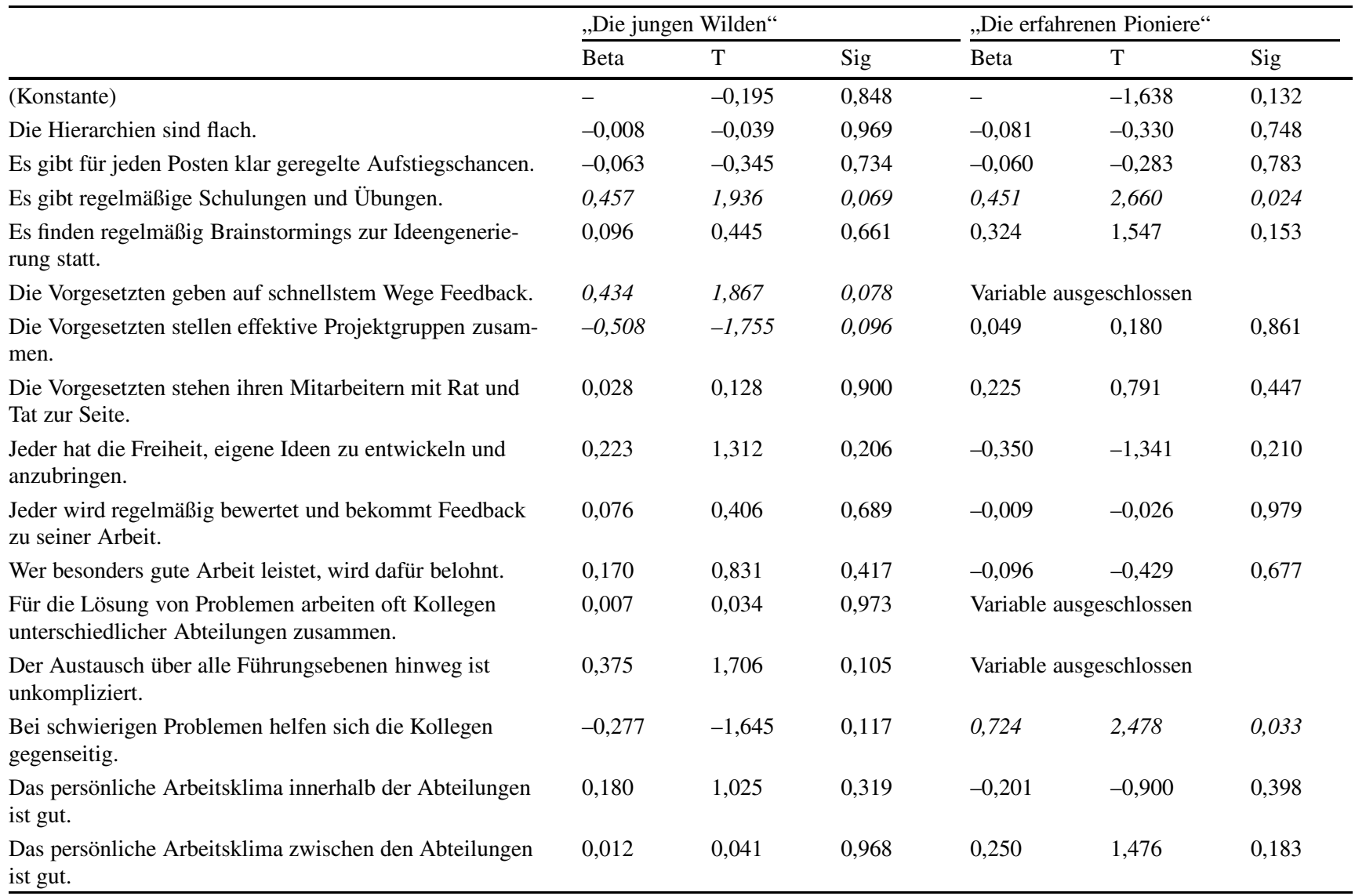

Signifikante Zusammenhänge mit $p<0,100$ sind kursiv markiert

onsfernen Branchen trotzdem innovativ, auch wenn keine innovationsfreundliche Unternehmenskultur gelebt wird. Innovationsförderliche Angebote in der Arbeitsstrukturierung hingegen besitzen einen kleinen direkten Einfluss auf innovative Verhaltensweisen und beeinflussen zudem positiv, ob aus einem individuellen Kreativitätspotenzial innovatives Verhalten wird. Diese Zusammenhänge haben sich als altersunabhängig herausgestellt.

Als weiterer Treiber innovativen Verhaltens konnte die Zufriedenheit mit der selbstempfundenen Ausschöpfung des eigenen Potenzials im Arbeitsumfeld ausgemacht werden. Diese Ausschöpfungszufriedenheit hat einen kleinen direkten Effekt auf das innovative Verhalten, der noch stärker wird, wenn das Kreativitätspotenzial in die Einflussgleichung mit aufgenommen wird. Wenn Menschen mit hohem Kreativitätspotenzial eine hohe Ausschöpfungszufriedenheit empfinden, führt dies zu deutlich innovativerem Verhalten im Arbeitsumfeld.

Ein Blick in die Treiber der Ausschöpfungszufriedenheit zeigt, dass unabhängig vom Alter der Befragten regelmäßige Schulungen und Übungen zu mehr Zufriedenheit führen. Allerdings erwarten junge Innovative (,die jungen Wilden“) viel mehr Führung und Unterstützung als ältere Innovative (,die erfahrenen Pioniere“). Dies ist insofern überraschend, als die sog. Generation Y als sehr freiheitsliebend und mit starkem Fokus auf individuelle Selbstverwirklichung verbunden wird (Klös et al. 2016, S. 20ff.), lässt sich aber mit dem mangelnden Erfahrungsschatz dennoch verargumentieren. Gespiegelt wird diese Suche nach Sicherheit und Anleitung bei gleichzeitigem Drang nach Individualität auch in der verstärkten Nutzung verschiedenster Komponenten der sog. New Work, die gleichermaßen flexible Strukturen, aber auch Austauschformate und Rahmenkonstrukte für Innovationen (Ideenmanagement, Kreativräume) umfassen. Die ,erfahrenen Pioniere“ hingegen brauchen in erster Linie Flächen und Strukturen für interpersonellen Austausch, was sich mit der theoretischen Annahme deckt, dass mit steigendem Alter das Erfahrungswissen nur durch kollaborativen Austausch zu innovativen Verhaltensweisen führt (Noefer et al. 2009).

Insgesamt hat sich gezeigt, dass Befragte aus eher innovationsfernen Branchen ein anderes Einflussmuster bei innovativem Verhalten zu zeigen scheinen, als dies in Branchen der Fall ist, in denen Innovationen bereits zur Unter- 
Tab.7 Clusterspezifische Korrelation (Pearson) zwischen Einzelitems der innovationsförderlichen Unternehmensstruktur und Ausschöpfungszufriedenheit. (Quelle: Eigene Darstellung)

\begin{tabular}{|c|c|c|c|c|}
\hline & \multicolumn{2}{|c|}{ „Die jungen Wilden“ } & \multicolumn{2}{|c|}{ „Die erfahrenen Pioniere“ } \\
\hline & Korr. (Pearson) & Sign & Korr. (Pearson) & Sign \\
\hline Desk Sharing & $-0,032$ & 0,884 & 0,220 & 0,431 \\
\hline Flächen für gegenseitige Begegnung und Austausch & 0,200 & 0,317 & 0,564 & 0,018 \\
\hline Gaming-Ecken & $-0,296$ & 0,325 & 0,149 & 0,662 \\
\hline Mobiles Arbeiten & 0,166 & 0,347 & 0,068 & 0,769 \\
\hline Ideenmanagement & 0,413 & 0,032 & 0,334 & 0,175 \\
\hline Co-Working-Bereiche & 0,204 & 0,416 & $-0,067$ & 0,812 \\
\hline Flächen für spontanen, informellen Austausch & 0,220 & 0,212 & 0,243 & 0,289 \\
\hline Flächen mit Kreativmaterialien für Projektteams & 0,372 & 0,067 & $-0,061$ & 0,804 \\
\hline Flächen für Ruhe und Entspannung & 0,094 & 0,692 & $-0,163$ & 0,596 \\
\hline Flächen für individuelle Bewegung & 0,246 & 0,362 & 0,065 & 0,834 \\
\hline Virtuelle Kollaborations-Instrumente & 0,130 & 0,487 & 0,264 & 0,291 \\
\hline $\begin{array}{l}\text { Kampagnen zur Ideenfindung und -entwicklung über digitale } \\
\text { Plattformen }\end{array}$ & 0,372 & 0,097 & 0,317 & 0,250 \\
\hline Abteilungsübergreifende Vernetzungsveranstaltungen & 0,163 & 0,459 & 0,548 & 0,012 \\
\hline Abteilungsinterne informelle Austauschformate & 0,381 & 0,029 & 0,499 & 0,030 \\
\hline $\begin{array}{l}\text { Förderung der Teilnahme an externen Netzwerkveranstaltun- } \\
\text { gen }\end{array}$ & 0,491 & 0,011 & 0,189 & 0,451 \\
\hline Förderung von Selbstorganisation im Unternehmen & 0,531 & 0,006 & 0,254 & 0,293 \\
\hline Anteile frei nutzbarer Arbeitszeit für kreative Projekte & 0,601 & 0,014 & $-0,257$ & 0,445 \\
\hline Sport- und Freizeitangebote & $-0,198$ & 0,322 & 0,188 & 0,456 \\
\hline Feelgood-Management & $-0,045$ & 0,889 & $-0,168$ & 0,621 \\
\hline Weiterbildung & 0,257 & 0,142 & 0,189 & 0,389 \\
\hline
\end{tabular}

Signifikante Zusammenhänge mit $p<0,100$ sind kursiv markiert

Tab. 8 Überblick über die Ergebnisse der Hypothesenprüfung. (Quelle: Eigene Darstellung)

\begin{tabular}{ll}
\hline H-F1-a & Je höher das Kreativitätspotenzial von Mitarbeitenden ist, desto stärker zeigen sie innovative Verhaltensweisen. \\
H-F1-b & $\begin{array}{l}\text { Trifft ein hohes Kreativitätspotenzial auf eine innovationsfreundliche Unternehmenskultur, hat dies einen positiven } \\
\text { Einfluss auf innovative Verhaltensweisen. } \\
\text { H-F1-c }\end{array}$ \\
& $\begin{array}{l}\text { Trifft ein hohes Kreativitätspotenzial auf eine innovationsförderliche Unternehmensstruktur, hat dies einen positiven } \\
\text { Einfluss auf innovative Verhaltensweisen. }\end{array}$ \\
H-F1-d & $\begin{array}{l}\text { Wie stark innovationsfreundliche Unternehmenskultur und innovationsförderliche Unternehmensstruktur zu einer } \\
\text { Ausschöpfung des individuellen Kreativitätspotenzials führen, hängt vom Alter des Mitarbeitenden ab. }\end{array}$ \\
H-F2-a & $\begin{array}{l}\text { Je höher die Zufriedenheit mit der Ausschöpfung der eigenen Innovativität (Ausschöpfungszufriedenheit) einge- } \\
\text { schätzt wird, desto stärker zeigen Mitarbeitende innovative Verhaltensweisen. }\end{array}$ \\
H-F2-b & $\begin{array}{l}\text { Je besser innovationsfreundliche Unternehmenskultur und innovationsförderliche Unternehmensstruktur das indivi- } \\
\text { duelle Kreativitätspotenzial ausschöpfen, desto höher ist auch die empfundene Ausschöpfungszufriedenheit. } \\
\text { Die Ausschöpfungszufriedenheit beeinflusst den Effekt, den innovationsfreundliche Unternehmenskultur und in- } \\
\text { H-F2-c }\end{array} \quad \begin{array}{l}\boldsymbol{x} \\
\text { novationsförderliche Unternehmensstruktur bei der Überführung bestehenden Kreativitätspotenzials in innovatives }\end{array}$ \\
\hline
\end{tabular}

nehmens-DNA zählen. Bei der Interpretation der vorliegenden Daten ist jedoch zu berücksichtigen, dass die Stichprobengröße recht klein, nicht repräsentativ und in sich recht homogen war. Die Ergebnisse ermutigen jedoch, sich in umfassenderen Studien mit branchen-, aber auch altersabhängigen Treibern von innovativem Verhalten im Arbeitsumfeld weiter zu beschäftigen. Tab. 8 zeigt noch einmal die Ergebnisse der Hypothesenprüfung im Überblick.

\section{Praktische Relevanz}

Was können Unternehmen aus innovationsfernen Branchen konkret tun, um einerseits innovatives Verhalten zu fördern (F1) und andererseits Zufriedenheit und auch Bindung der Mitarbeitenden zu erhöhen (F2)?

Strukturen schaffen und nach außen kommunizieren. Da den Ergebnissen zufolge das individuelle Kreativitätspotenzial einen Einfluss auf innovatives Verhalten im 
Tab.9 Mittelwertvergleich des semantischen Differenzials von strukturellen Gegebenheiten im Arbeitsumfeld (Hays et al. 2016) im Vergleich zwischen mittlerem und sehr hohem Kreativitätspotenzial. (Quelle: Eigene Darstellung)

\begin{tabular}{|c|c|c|c|c|c|c|}
\hline & \multirow[b]{2}{*}{ Semantisches Differenzial } & \multicolumn{2}{|c|}{$\begin{array}{l}\text { Mittleres Kreativitäts- } \\
\text { potenzial }(N=21)\end{array}$} & \multicolumn{2}{|c|}{$\begin{array}{l}\text { Sehr hohes Kreativi- } \\
\text { tätspotenzial }(N=3)\end{array}$} & \multirow{2}{*}{$\begin{array}{l}\text { Signifikanz des } \\
\text { MW-Vergleichs } \\
\text { (t-Test) }\end{array}$} \\
\hline & & MW & SD & MW & SD & \\
\hline $\begin{array}{l}\text { Einflussnahme auf eigene Ar- } \\
\text { beitsbedingungen (Zeit, Ort) }\end{array}$ & $\begin{array}{l}\text { Fremdbestimmt (1) } \\
\text { Selbstbestimmt (5) }\end{array}$ & 5,05 & 0,865 & 5,33 & 0,577 & 0,503 \\
\hline Führungskultur & $\begin{array}{l}\text { Auf Kontrolle basierend (1) } \\
\text { Auf Vertrauen basierend ( } 5 \text { ) }\end{array}$ & 4,67 & 1,017 & 6,00 & 0,000 & 0,000 \\
\hline $\begin{array}{l}\text { Anpassungsdruck (Erschei- } \\
\text { nungsbild, Arbeitsstil) }\end{array}$ & $\begin{array}{l}\text { Druck, sich anzupassen (1) } \\
\text { Freiheit, man selbst zu sein (5) }\end{array}$ & 4,76 & 0,944 & 5,67 & 0,577 & 0,086 \\
\hline Organisationsstruktur & $\begin{array}{l}\text { Bürokratisch, zentralisiert (1) } \\
\text { Unternehmerisch, flexibel (5) }\end{array}$ & 4,24 & 1,411 & 5,33 & 0,577 & 0,050 \\
\hline Experimentierkultur & $\begin{array}{l}\text { Vermeiden von Fehlern (1) } \\
\text { Lernen aus Fehlern (5) }\end{array}$ & 4,62 & 0,308 & 5,67 & 0,577 & 0,068 \\
\hline $\begin{array}{l}\text { Einflussnahme auf Unterneh- } \\
\text { mensentscheidungen }\end{array}$ & $\begin{array}{l}\text { Hierarchisch (1) } \\
\text { Demokratisch (5) }\end{array}$ & $\mathbf{3 , 5 7}$ & 1,568 & 5,00 & 1,000 & 0,108 \\
\hline
\end{tabular}

Signifikante Mittelwertunterschiede mit $p<0,100$ sind kursiv markiert

Arbeitsumfeld hat, ist es für Unternehmen aus innovationsfernen Branchen empfehlenswert, Personen mit hohem Kreativitätspotenzial durch entsprechende, für diese Personengruppe attraktive Strukturen anzuziehen. Dabei bevorzugen Mitarbeitende mit sehr hohem im Vergleich zu Mitarbeitenden mit mittlerem Kreativitätspotenzial unternehmensstrukturelle und -kulturelle Gegebenheiten, die stärker auf eine vertrauensbasierte Führungskultur, individuelle Freiheit, flexible Organisationsstrukturen und eine positive Fehlerkultur fokussieren (Tab. 9). Mittels eines derart ausgestalteten Arbeitsumfelds wird es Mitarbeitenden ermöglicht, ihr Kreativitätspotenzial ausschöpfen und für das Unternehmen gewinnbringend nutzen zu können.

\section{Strukturelle Angebote sind innovationsförderlicher als der komplizierte Umbau einer Unternehmenskultur. Wie die} Ergebnisse gezeigt haben, führt das Vorhandensein konkreter Angebote der Innovationsförderung altersunabhängig zu innovativem Verhalten, und zwar tatsächlich unabhängig von der (nicht) vorhandenen innovationsfreundlichen Unternehmenskultur. Dies ist für Unternehmen in eher innovationsfernen Branchen ein ermutigendes Ergebnis, weil strukturelle Änderungen in Richtung Innovationsförderung deutlich einfacher und schneller umsetzbar sind als Änderungen der Unternehmenskultur.

Altersabhängig fördern, um Mitarbeiter zu binden. Weil Zufriedenheit im Arbeitsumfeld eine wichtige Basis für eine langfristige Mitarbeitendenbindung darstellt, ist es erfreulich, dass die Ausschöpfungszufriedenheit zusätzlich einen Beitrag zur Innovativität der Belegschaft beisteuert. Sie lässt sich allerdings nicht über Altersstufen hinweg gleichermaßen erreichen. Ein internes Weiterbildungsmanagement fördert gemäß den gegebenen Fähigkeiten die Ausschöpfungszufriedenheit altersunabhängig. Jüngere Mitar- beitende benötigen jedoch daneben mehr Unterstützung und Strukturen als ältere Mitarbeitende und empfinden ihr eigenes Kreativitätspotenzial dann als ausgeschöpft, wenn sie ein breites Spektrum an Angeboten von New Work nutzen können, von der flexiblen Arbeitsorganisation bis hin zu Methoden und Strukturen des Ideenmanagements. Um bei älteren innovativen Mitarbeitenden eine höhere Ausschöpfungszufriedenheit zu erreichen, ist die Bereitstellung von unternehmensinternen und -externen Austauschformaten zwingend erforderlich, damit sie ihr Erfahrungswissen in assoziative Prozesse einbringen können.

Danksagung Wir danken der LF-Gruppe und insbesondere den Versicherungsforen Leipzig, den Energieforen Leipzig sowie den Digital Impact Labs Leipzig für die Unterstützung dieser Studie.

Funding Open Access funding enabled and organized by Projekt DEAL.

Open Access Dieser Artikel wird unter der Creative Commons Namensnennung 4.0 International Lizenz veröffentlicht, welche die Nutzung, Vervielfältigung, Bearbeitung, Verbreitung und Wiedergabe in jeglichem Medium und Format erlaubt, sofern Sie den/die ursprünglichen Autor(en) und die Quelle ordnungsgemäß nennen, einen Link zur Creative Commons Lizenz beifügen und angeben, ob Änderungen vorgenommen wurden.

Die in diesem Artikel enthaltenen Bilder und sonstiges Drittmaterial unterliegen ebenfalls der genannten Creative Commons Lizenz, sofern sich aus der Abbildungslegende nichts anderes ergibt. Sofern das betreffende Material nicht unter der genannten Creative Commons Lizenz steht und die betreffende Handlung nicht nach gesetzlichen Vorschriften erlaubt ist, ist für die oben aufgeführten Weiterverwendungen des Materials die Einwilligung des jeweiligen Rechteinhabers einzuholen.

Weitere Details zur Lizenz entnehmen Sie bitte der Lizenzinformation auf http://creativecommons.org/licenses/by/4.0/deed.de. 


\section{Literatur}

Amabile, T. M. (1988). A model of creativity and innovation in organizations. Research in Organizational Behavior, 10(1), 123-167.

Amabile, T. M., Conti, R., Coon, H., Lazenby, J., \& Herron, M. (1996). Assessing the Work Environment for Creativity. The Academy of Management Journal, 39(5), 1154-1184. https://doi.org/10.5465/ 256995.

Arbeitgeberverband der Versicherungsunternehmen (AGV) (2020). Der Branchensozialreport 2020. https://www.agv-vers.de/file

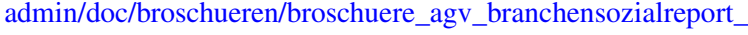
2020.pdf. Zugegriffen: 19. Sept. 2021.

Arbeitgeberverband der Versicherungsunternehmen (AGV) (o.J.). Beschäftigtenstruktur/Altersstruktur. https://www.agv-vers.de/ statistiken/branchenzahlen/beschaeftigtenstruktur/altersstruktur. html. Zugegriffen: 19. Sept. 2021.

Bacher, J., Pöge, A., \& Wenzig, K. (2010). Clusteranalyse: Anwendungsorientierte Einführung in Klassifizierungsverfahren (3. Aufl.). München: Oldenbourg Verlag.

Baltes, P. B., et al. (1999). Lifespan Psychology: Theory and Application to Intellectual Functioning. Annual Review of Psychology, 50, 471-507. https://doi.org/10.1146/annurev.psych.50.1.471.

Baron, R. M., \& Kenny, D. A. (1986). The moderator-mediator variable distinction in social psychological research: Conceptual, strategic, and statistical considerations. Journal of Personality and Social Psychology, 51(6), 1173-1182. https://doi.org/10.1037/00223514.51.6.1173.

Barsade, S. G., \& Gibson, D.E. (1998). Group emotion: A view from top and bottom. Research on Managing Groups and Teams, 1998(1), 81-102.

Bergmann, F. (2004). Neue Arbeit, Neue Kultur. Freiburg im Breisgau: Arbor.

BIB (2019). Alterung und Arbeitsmarkt: Auswirkungen weniger dramatisch als vielfach befürchtet. Wiesbaden: Bundesinstitut für Bevölkerungsforschung (BIB).

BMBF (Hrsg.). (2020). Daten und Fakten zum deutschen Forschungsund Innovationssystem. Berlin: Bundesministerium für Bildung und Forschung.

Brem, A. (2016). Wie kann man Kreativität in Unternehmen stimulieren?: Individuelle, gruppenspezifische und unternehmensweite Kreativität im Ideen- und Innovationsmanagement. Ideen- und Innovationsmanagement, 42(3), 87-90. https://doi.org/10.37307/ j.2198-3151.2016.03.03.

BWG AG St. Galler Innovationskultur Navigator. https://innovationskul turnavigator.ch/6-dimensionen-der-unternehmenskultur. Zugegriffen: 19. Sept. 2021.

Carayannis, E. G., \& Gonzalez, E. (2003). Creativity and innovation = competitiveness? When, how, and why. In L. V. Shavinina (Hrsg.), The international handbook on innovation (S. 587-605). Oxford: Pergamon.

Collins, M.A., \& Amabile, T.M. (2006). Motivation and Creativity. In R. J. Sternberg (Hrsg.), Handbook of Creativity (9. Aufl. S. 297-312). Cambridge: Cambridge University Press.

Cook, C., Heath, F., \& Thompson, R.L. (2000). A meta-analysis of response rates in web- or internet-based surveys. Educational and Psychological Measurement, 60(6), 821-836. https://doi.org/10. 1177/00131640021970934.

Csikszentmihalyi, M. (1988). Motivation and creativity: Towards a synthesis of structural and energistic approaches to cognition. New Ideas in Psychology, 6(2), 159-176. https://doi.org/10.1016/ 0732-118X(88)90001-3.

Disselkamp, M. (2012). Innovationsmanagement. Instrumente und Methoden zur Umsetzung in Unternehmen. Wiesbaden: Springer Gabler. https://doi.org/10.1007/978-3-8349-4472-6.

Edmondson, A.C. (2018). The fearless organization: creating psychological safety in the workplace for learning, innovation, and growth. : John Wiley \& Sons.
Eisenberger, R., \& Cameron, J. (1996). Detrimental effects of reward: reality or myth? American Psychologist, 51(1), 1153-1166. https://doi.org/10.1037/0003-066X.51.11.1153.

Ernst, H. (2003). Unternehmenskultur und Innovationserfolg - Eine empirische Analyse. Schmalenbachs Zeitschrift für betriebswirtschaftliche Forschung, 55(1), 23-44. https://doi.org/10.1007/ BF03372697.

Europäische Kommission (2015). Benutzerleitfaden zur Definition von KMU. Luxemburg: Amt für Veröffentlichungen der Europäischen Union. https://publications.europa.eu/resource/cellar/79c0ce87f4dc-11e6-8a35-01aa75ed71a1.0004.01/DOC_1. Zugegriffen: 23. Aug. 2021.

Fuglsang, L. (2008). Innovation with care-what it means. In L. Fuglsang (Hrsg.), Innovation and the creative process-towards innovation with care (S. 3-21). Cheltenham/Northampton: Edward Elgar Publishing.

GDV (Hrsg.). (2020). Statistisches Taschenbuch der Versicherungswirtschaft 2020. Berlin: Gesamtverband der Deutschen Versicherungswirtschaft e. V. (GDV).

Geis-Thöne, W. (2021). IW-Report 11/2021 - Mögliche Entwicklungen des Fachkräfteangebots bis zum Jahr 2040. Eine Betrachtung der zentralen Determinanten und Vorausberechnungen. Köln: Institut der deutschen Wirtschaft. https://www.iwkoeln. de/fileadmin/user_upload/Studien/Report/PDF/2021/IW-Report_ 2021-Fachkraefteangebot-2040.pdf. Zugegriffen: 26. Sept. 2021.

Gough, H.G. (1979). A creative personality scale for the adjective check list. Journal of Personality and Social Psychology, 37(8), 1398-1405. https://doi.org/10.1037/0022-3514.37.8.1398.

Grüling, B. (2019). Neue Arbeitswelt: Wir haben die Freiheit. In Frankfurter Allgemeine Zeitung (Hrsg.). New Work: Die Zukunft der Arbeit (S. 9-11). Verlagsspezial. Frankfurt: FAZ.

Hackl, B., Wagner, M., Attmer, L., \& Baumann, D. (2017). New Work: Auf dem Weg zur neuen Arbeitswelt. Management-Impulse, Praxisbeispiele, Studien. Wiesbaden: Springer. https://doi.org/10. 1007/978-3-658-16266-5.

Hayes, A.F. (2018). Introduction to mediation, moderation, and conditional process analysis. A regression-based-approach (3. Aufl.). New York, London: Guilford.

Hays, ZAAG, \& GfWM (Hrsg.). (2016). Der Ruf nach Freiheit: Innovationsförderliche Arbeitswelten aus Sicht der Arbeitenden. München: Hays. https://www.hays.de/documents/10192/118775/ studie-freiheit-sicherheit.pdf. Zugegriffen: 18. Juni 2021.

Higgins, J.M., \& Wiese, G.G. (1996). Innovationsmanagement Kreativitätstechniken für den unternehmerischen Erfolg. Berlin, Heidelberg: Springer. https://doi.org/10.1007/978-3-642-614316.

Janssen, O., \& Van Yperen, N.W. (2004). Employees' goal orienations, the quality of leader-member-exchange, and the outcomes of job performance and job satisfaction. Academy of Management Journal, 47(3), 368-384. https://doi.org/10.2307/20159587.

Judge, T. A., \& Ilies, R. (2004). Affect and job satisfaction-A study of their relationship at work and at home. Journal of Applied Psychology, 89(4), 661-673. https://doi.org/10.1037/0021-9010.89. 4.661 .

Kästner, E. (2009). Kreativität als Bestandteil der Markenidentität: Ein verhaltenstheoretischer Ansatz, zur Analyse der Mitarbeiterkreativität. Wiesbaden: Gabler. https://doi.org/10.1007/978-38349-8452-4.

Kleinginna, P.R., \& Kleinginna, A. M. (1981). A categorized list of emotion definitions with a suggestion for a consensual definition. Motivation and Emotion, 5(4), 345-379. https://doi.org/10.1007/ BF00992553.

Klös, H.-P., Rump, J., \& Zibrowius, M. (2016). Die neue Generation: Werte, Einstellungen und unternehmerische Anforderungen. Roman Herzog Institut, Arbeitspapier Nr. 29. München: Roman Herzog Institut.

Melde, A., \& Benz, M. (2014). Employer Branding in Wissenschaft und Praxis: Wie mittelständische Unternehmen ihre Arbeitgeber- 
positionierung international erfolgreich gestalten können. Leipzig: Fraunhofer MOEZ.

Noefer, K., Stegmaier, R., Molter, B., \& Sonntag, K. (2009). Innovatives Verhalten über die Altersspanne: Effekte von Feedback, Unterstützung der horizontalen Mobilität und entwicklungsbezogener Selbstwirksamkeit. Zeitschrift für Personalpsychologie, 8(2), 47-58. https://doi.org/10.1026/1617-6391.8.2.47.

Oldham, G. R., \& Cummings, A. (1996). Employee creativity: personal and contextual factors at work. Academy of Management Journal, 39(3), 607-634. https://doi.org/10.2307/256657.

Preuß, O. (2021). Wir laufen auf einen gravierenden Fachkräftemangel zu. In Die WELT vom 19.03.2021. https://www.welt.de/ regionales/hamburg/article228725057/Energiewende-und-ITWir-laufen-auf-einen-gravierenden-Fachkraeftemangel-zu.html. Zugegriffen: 19. Sept. 2021.

Pritschet, L., Powell, D., \& Horne, Z. (2016). Marginally significant effects as evidence for hypotheses: changing attitudes over four decades. Psychological Science, 27(7), 1036-1042. https://doi.org/ 10.1177/0956797616645672.

Rammer, C. (2021). Deutsche Wirtschaft bleibt trotz Corona-Pandemie auf Innovationskurs. ZEW News, März 2021, 6-7.

Reischies, F.M., \& Lindenberger, U. (2010). Grenzen und Potentiale kognitiver Leistungsfähigkeit im Alter. In U. Lindenberger, J. Smith, K. U. Mayer \& P. B. Baltes (Hrsg.), Die Berliner Altersstudie (3. Aufl. S. 375-400). Berlin: Oldenbourg Akademie.

Ruef, M. (2002). Strong ties, weak ties and islands: structural and cultural predictors of organizational innovation. Industrial and Corporate Change, 11(3), 427-449. https://doi.org/10.1093/icc/11.3. 427.

Sarges, W. (2013). Management-Diagnostik. Göttingen: Hogrefe.

Segler, T. (2000). Kreativitätsförderung im Unternehmen. In R. M. Holm-Hadulla (Hrsg.), Heidelberger Jahrbücher. Kreativität, (Bd. 44, S. 77-108). Berlin, Heidelberg: Springer. https:// doi.org/10.1007/978-3-642-87237-2_6.

Shalley, C.E. (1995). Effects of coactions, expected evaluation, and goal setting on creativity and productivity. Academy of Management Journal, 38(2), 483-503. https://doi.org/10.2307/256689.

Shalley, C.E., et al. (2000). Matching creativity requirements and the work environment: effects on satisfaction and intention to leave. Academy of Management Journal, 43(2), 215-223. https://doi. org/10.5465/1556378.

Statistisches Bundesamt (2021). Pressemitteilung Nr. 164 vom 1. April 2021. https://www.destatis.de/DE/Presse/Pressemitteilungen/ 2021/04/PD21_164_431.html. Zugegriffen: 19. Sept. 2021.

Statistisches Bundesamt (2021a). Energie: Beschäftigte, Umsatz, Investitionen. https://www.destatis.de/DE/Themen/BranchenUnternehmen/Energie/Beschaeftigte-Umsatz-Investitionen/ inhalt.html\#sprg236398. Zugegriffen: 19. Sept. 2021.

Volkholz, V. (2005). Kreativität und Innovativität Älterer. Dortmund: Gesellschaft für Arbeitsschutz- und Humanisierungsforschung.
Winkelhofer, G. (2006). Kreativ managen. Ein Leitfaden für Unternehmer, Manager und Projektleiter. New York: Springer.

Woodman, R. W., et al. (1993). Toward a theory of organizational creativity. Academy of Management Review, 18(2), 293-321. https:// doi.org/10.2307/258761.

ZEW (Hrsg.). (2021). Innovationen in der deutschen Wirtschaft. Indikatorenbericht zur Innovationserhebung 2020., Mannheim: ZEW - Leibniz-Zentrum für Europäische Wirtschaftsforschung. https:// ftp.zew.de/pub/zew-docs/mip/20/mip_2020.pdf?v=1616141836. Zugegriffen: 19. Sept. 2021.

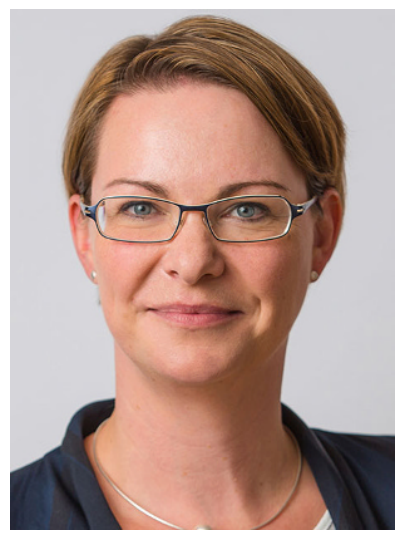

Prof. Dr. Evelyn Kästner ist Professorin für Medienmanagement und Marketing an der Hochschule Macromedia. Als studierte Kommunikations- und Medienwissenschaftlerin und promovierte Marketingwissenschaftlerin forschte sie mehrere Jahre lang zu Markenführung, Medienmanagement und Innovationsmanagement. Ihre beruflichen Stationen führten sie als Marketing-, Vertriebs- und Kommunikationsleiterin in die Bereiche Biomedizin, Weiterbildung und Personaldienstleistungen. Vor ihrem Wechsel in die akademische Welt im November 2019 war sie zuletzt Pressesprecherin und Kommunikationsleiterin an einem biomedizinischen Leibniz-Institut.

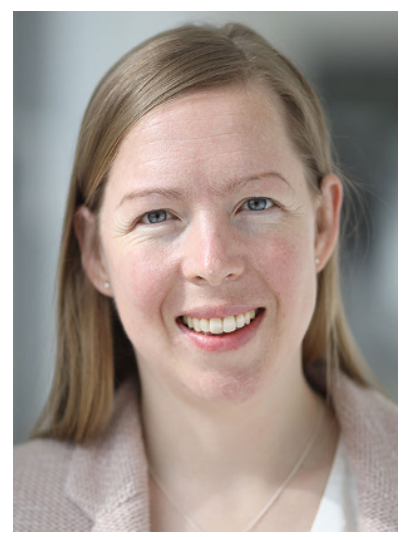

Prof. Dr. Katja Rudolph ist Professorin für Allgemeine Betriebswirtschaftslehre, Marketing und EBusiness an der Hochschule Merseburg. Der Schwerpunkt ihrer Forschung liegt an der Schnittstelle von Konsumentenverhaltensforschung und den Bereichen Innovation und Nachhaltigkeitsmarketing. Zuvor war sie bei den Digital Impact Labs Leipzig tätig und setzte sich u.a. in Forschungs- und Praxisprojekten mit dem Thema New Work und der Veränderung der Arbeitswelten insbesondere im Innovationskontext auseinander. 